\title{
Growth of graphitic carbon layers around silicon carbide nanowires
}

Neeraj Mishra ${ }^{1}$, Matteo Bosi ${ }^{2}$, Francesca Rossi ${ }^{2}$, Giancarlo Salviati ${ }^{2}$, John Boeckl ${ }^{3}$, Francesca Iacopi $^{1, a)}$

${ }^{1}$ School of Electrical and Data Engineering, University of Technology Sydney, Broadway, 2007 NSW, Australia

${ }^{2}$ IMEM-CNR Institute, Parco Area delle Scienze 37/A, 43124 Parma, Italy

${ }^{3}$ Materials and Manufacturing Directorate, Air Force Research Laboratories, WrightPatterson AFB, $45433 \mathrm{OH}, \mathrm{USA}$

a) $\underline{\text { rancesca.iacopi@uts.edu.au }}$

\begin{abstract}
We demonstrate the ability to synthesize graphitic carbon sheets around cubic silicon carbide nanowires via an alloy-mediated catalytic process. The transmission electron microscopy analysis shows multilayer graphitic carbon sheets with a large interatomic layer distance of $\sim 0.45 \mathrm{~nm}$, suggesting the presence of oxygen in the graphitic system. Oxygenrelated peaks observed by energy-dispersive X-ray spectroscopy, Raman spectroscopy and Fourier-transform infrared spectroscopy further confirm the oxidation of the graphitic carbon layers. A detailed investigation of the Raman spectra reveals a turbostratic stacking of the graphitic carbon layers. The turbostratic nature and the presence of oxidation in the graphitic carbon surrounding the silicon carbide nanowires makes them a suitable platform for further functionalisation, of particular interest for bio-sensing, as both graphitic carbon and silicon carbide are bio-compatible.
\end{abstract}




\section{INTRODUCTION}

Over the past decade, graphene, as well as its derivatives such as graphene oxide (GO) and reduced graphene oxide (rGO), have emerged as preferred material for bio-sensing applications ${ }^{1-3}$. This is partly due to their $2 \mathrm{D}$ nature as well as their superior chemical and mechanical stability properties ${ }^{2}$. In order to fabricate graphene-based biosensors, graphene is either transferred on a suitable substrate (for fabricating nanodevices such as transistors ${ }^{4}$ ) or mixed with other metal/semiconducting nanomaterials (nanoparticles, nanowires) made of gold $(\mathrm{Au})^{5,6}$, silver $(\mathrm{Ag})^{7}$, silicon carbide $(\mathrm{SiC})^{8-10}$ etc. Comprehensive reviews on graphene and graphene oxide -based biosensors are found in recent literature ${ }^{11-13}$.

In parallel, $\mathrm{SiC}$ nanostructures have shown potential towards chemical sensors ${ }^{14,15}$, transistors for deoxyribonucleic acid (DNA)/ribonucleic acid (RNA) detection ${ }^{16,}$ 17, photodynamic therapy ${ }^{18}$ and implantable scaffolds for tissue regeneration. ${ }^{19}$ The coupling of favourable electrical and optical/plasmonic properties of graphene with exceptional mechanical properties of $\mathrm{SiC}$ nanowires (NWs) could result in a hybrid biocompatible nanomaterial for next generation biosensors. ${ }^{9,10,13,20}$ Note that both graphene and SiC are biocompatible. Recently, researchers reported the synergistic effects of a nanocomposite made with SiC NWs and GO sheets/foam. ${ }^{9}, 10$ Their experimental results indicated that the nanocomposite improve adhesion/compatibility of the $\mathrm{SiC}$ with polymers ${ }^{10}$ as well as enhance the thermal stability and electromagnetic absorption of $\mathrm{rGO}$ foams ${ }^{9}$. Note that the abovementioned nanocomposites were obtained by either mixing GO with $\mathrm{SiC}$ NWs in bulk in a solution ${ }^{10}$ or by means of a vapour-solid reaction process $^{9}$. Such processes typically lack control at the nanoscale, leading among others to poor yield. Therefore, a direct method to grow graphitic layers on the SiC NWs would be highly desirable. In this manuscript, we demonstrate a method for direct growth of graphitic carbon (GC) sheets on cubic silicon carbide (3C-SiC) NWs, which is based on the 
learnings from our alloy-mediated catalytic approach to grow graphene on 3C-SiC epitaxial films on silicon substrates ${ }^{21-23}$.

\section{EXPERIMENTAL}

The $3 \mathrm{C}-\mathrm{SiC}$ NWs growth was performed at $1100^{\circ} \mathrm{C}$ in a chemical vapour deposition $(\mathrm{CVD})$ reactor on $\mathrm{Si}(100)$ substrates. Nickel nitrate $\left(\mathrm{Ni}\left(\mathrm{NO}_{3}\right)_{2}\right)$ was used as a catalyst and carbon monoxide $(\mathrm{CO})$ as a gaseous precursor. This procedure resulted in core/shell $\mathrm{SiC} / \mathrm{SiO}_{2}$ NWs with the detailed nanowire growth process reported elsewhere. ${ }^{24,25}$ The $\mathrm{SiO}_{2}$ shell was then chemically etched using a standard RCA clean followed by a second chemical treatment with a Piranha solution and a final etching with $\mathrm{HCl}: \mathrm{H}_{2} \mathrm{O}(2: 1)$ and $\mathrm{H}_{2} \mathrm{O}: \mathrm{HF}(50: 1)$.

We have modified a $\mathrm{Ni} / \mathrm{Cu}$ alloy-mediated catalytic approach to attempt the graphitization of the SiC NWs. We had previously developed this synthesis method to grow large -scale graphene on hetero-epitaxial $3 \mathrm{C}-\mathrm{SiC}$ films grown on silicon substrates ${ }^{21-23}$. The epitaxial graphene obtained through this method resulted in the best quality graphene grown to-date on 3C-SiC on silicon, thanks to its liquid phase epitaxial growth nature.

A schematic of the graphitization process sequence adopted for the NWs is shown in Fig. 1. First, a double layer of $\sim 5 \mathrm{~nm}$ thick nickel (Ni) and $\sim 10 \mathrm{~nm}$ thick copper $(\mathrm{Cu})$ was sputtered over the NWs in a custom -made vacuum chamber using a $100 \mathrm{~mA}$ deposition current under a $10^{-5}$ mbar base pressure. Subsequently, the alloy-mediated graphitization was performed in a high temperature, low vacuum tube furnace (Carbolite HT tube furnace), evacuated to a pressure below $5 \times 10^{-5}$ mbar. The NWs samples were annealed at $1050^{\circ} \mathrm{C}$ for one hour then left to cool to room temperature under vacuum. Finally, the graphitized NWs samples were immersed in a Freckle solution ${ }^{21}$ for 30 minutes, followed by rinsing with deionized water. This wet etching process assists with the removal of the intermixed layer of unreacted metal, metal silicides as well as of the excess carbon produced during the graphitization process. $^{21-23,26}$ 

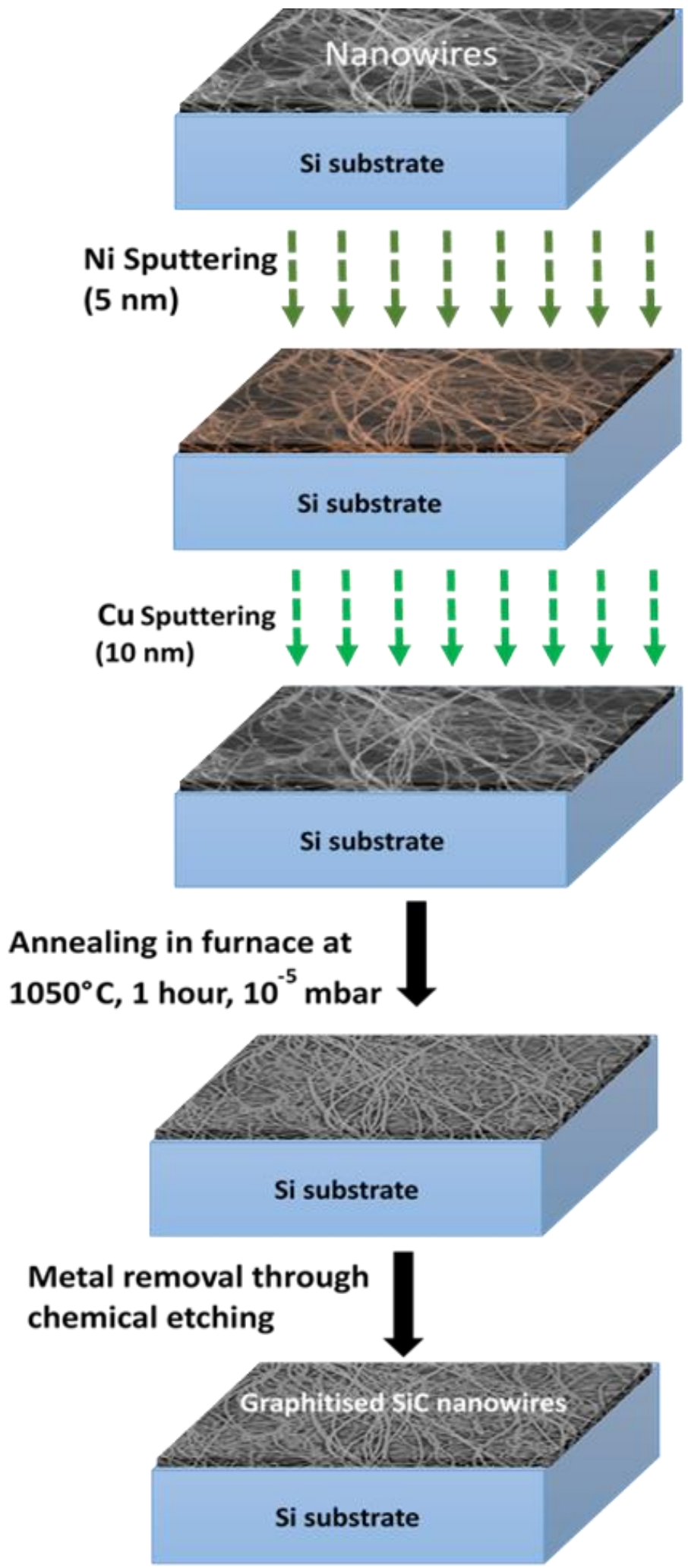

FIG. 1.Schematic of the alloy-mediated catalytic process for graphitizing $3 \mathrm{C}$-SiC nanowires grown on a silicon substrate.

The morphology of the NWs was characterized using a Zeiss Supra 55VP high-resolution fieldemission scanning electron microscope (FESEM) operating between 1-5 kV. An Oxford 
INCAx-sight energy-dispersive X-ray (EDX) spectroscopy attached to the FESEM was used to investigate the elemental composition of the NWs at $20 \mathrm{kV}$.

Raman spectroscopy was performed at room temperature using a Renishaw inVia Raman microscope equipped with argon-ion laser operating at $532 \mathrm{~nm}$ with a spot size of approximately $0.5 \mu \mathrm{m}^{2}$. The $\mathrm{D}, \mathrm{G}$ and $2 \mathrm{D}$ bands in the Raman spectrum of the graphitic layers were monitored and the intensity ratio (counts/second) of the D- to Gbands $\left(I_{\mathrm{D}} / I_{\mathrm{G}}\right)$ and $2 \mathrm{D}$ - to $\mathrm{G}$ - band $\left(I_{2 \mathrm{D}} / I_{\mathrm{G}}\right)$ was calculated. In order to improve the statistical accuracy of the $I_{\mathrm{D}} / I_{\mathrm{G}}$ and $I_{2 \mathrm{D}} / I_{\mathrm{G}}$ ratios, Raman spectra were recorded from ten different sites over the $2 \times 1 \mathrm{~cm}^{2}$ samples and averaged.

Attenuated Total Reflectance Fourier transformed infrared (ATR-FTIR) spectrum was measured with a Thermo Scientific Nicolet 6700 FTIR spectrometer in the range of 400$4000 \mathrm{~cm}^{-1}$ to identify the chemical bonds specific to the functional groups in the molecules.

Transmission Electron Microscopy (TEM) studies were performed using a JEOL JEM-2200 FS field-emission microscope, equipped with an in-column $\Omega$ filter. For observation, the NWs were transferred to carbon-coated copper grids by gentle rubbing. The microscope was operated at $200 \mathrm{kV}$, either in TEM mode to perform conventional and high-resolution imaging, or in scanning (STEM) mode to perform high angle annular dark field (HAADF) imaging and to acquire elemental maps by energy dispersive X-ray (EDX) spectroscopy. 


\section{RESULTS AND DISCUSSION}

\section{A. SEM/EDX}

In order to assess the alloy-mediated graphitization, we compared the surface morphology of the as-grown NWs with the graphitized NWs using a Zeiss Supra 55VP FESEM. The average diameter of the as -grown 3C-SiC NWs are in the range of 30-50 nm [Fig. 2(a)]. After the catalytic graphitization at $1050{ }^{\circ} \mathrm{C}$, some morphological changes are observed in particular a number of small granular asperities appear on the NWs surface [Fig. 2(c)].

EDX spectra [Fig. 2(b)] indicates a significant amount of silicon ( 45 weight $\%)$, carbon ( 25 weight $\%)$ and oxygen ( 29 weight $\%)$ present in the 3C-SiC NWs before graphitization. Although the silicon concentration remains almost similar ( 47 weight \%), a significant change in carbon and oxygen concentration was observed after graphitization [Fig. 2(d)]. The carbon concentration decreases significantly to $\sim 6$ weight $\%$. This is likely due to the consumption of the SiC NWs during the graphitization process, with the excess carbon released in the system being subsequently removed by the wet etch process. Note that the Freckle etch process was effective in removing all $\mathrm{Cu}$ residue while leaving minor clusters of $\mathrm{Ni}(\sim 5$ weight $\%)$. Since the NWs are only weakly attached to the Si surface, we have had to limit -our wet etch step duration to half an hour as opposed to the typical $12 \mathrm{~h}$ of the process used on flat $\mathrm{SiC}$ surfaces ${ }^{21-}$ ${ }^{23}$, limiting the removal efficiency of the metal residues. 

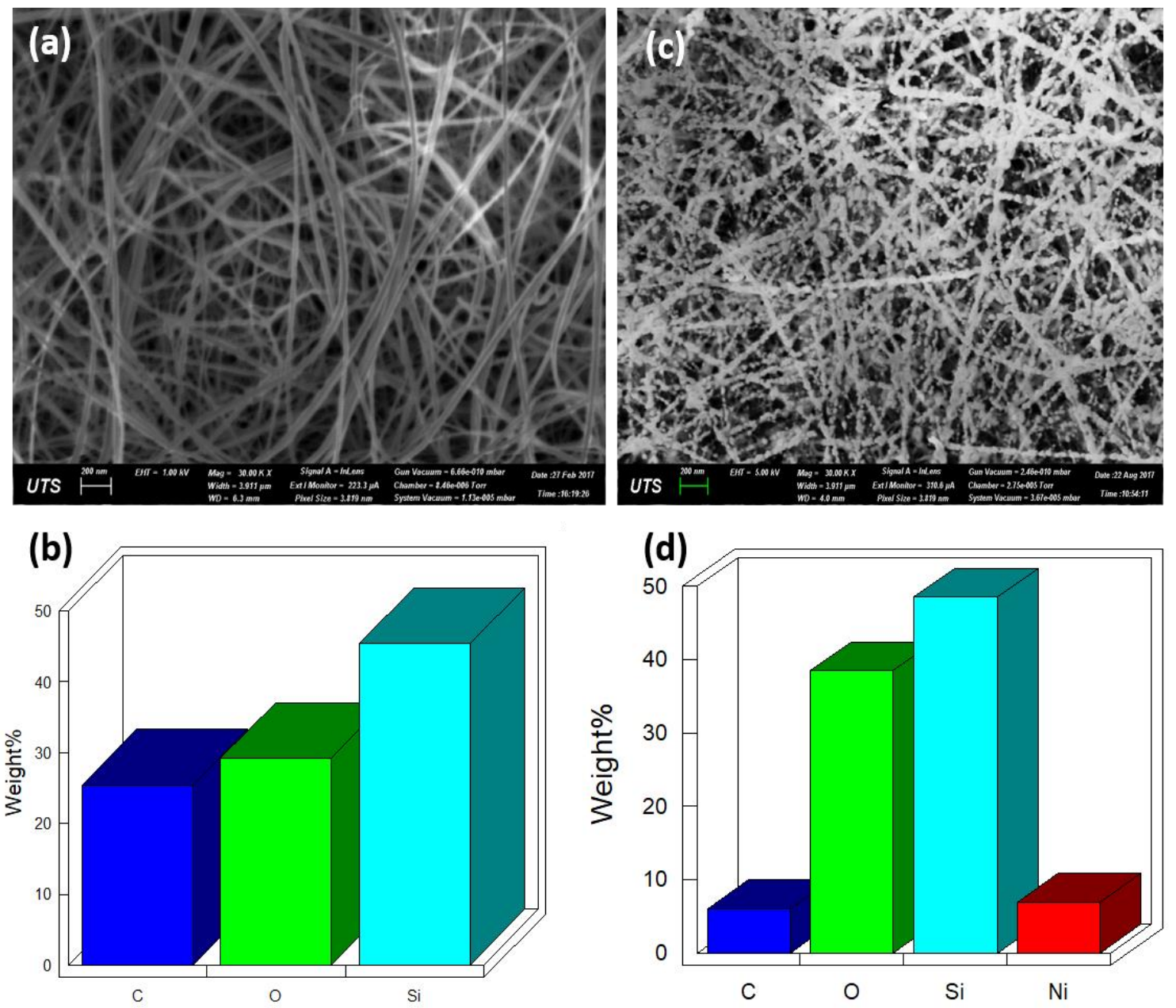

FIG. 2. SEM images of a) as-grown, and c) graphitized 3C-SiC NWs, where the scale bar in the micrographs corresponds to $200 \mathrm{~nm}$. The morphology of the SiC NWs after graphitization shows some granular surface asperities. b) and d) display EDX measurement results of as-grown and graphitized 3C-SiC NWs samples, respectively.

\section{B. Raman Spectroscopy}

Figure 3(a) shows the Raman spectra of an as-grown 3C-SiC NWs. The features observed between 900 and $1100 \mathrm{~cm}^{-1}$ are associated with second-order Raman scattering from the Si substrate $^{27}$. The characteristic transverse optical (TO) and longitudinal optical (LO) phonon modes are observed at $794 \mathrm{~cm}^{-1}$ and $973 \mathrm{~cm}^{-1}$, respectively. This confirms that the $\mathrm{SiC}$ NWs used for this work are cubic in nature ${ }^{28,29}$.

Raman spectra of the graphitized NWs [Fig. 3(b)] show the presence of the D, G and 2D bands at $\sim 1340 \mathrm{~cm}^{-1}, 1578 \mathrm{~cm}^{-1}$ and $2684 \mathrm{~cm}^{-1}$, respectively. Note that the displayed curve represents 
an average of 10 measured sites. The average $I_{\mathrm{D}} / I_{\mathrm{G}}$ ratio is about $1.3 \pm 0.1$, indicative of highly defective GC layers. Although we do not have directly relevant literature to compare to, the $I_{\mathrm{D}} / I_{\mathrm{G}}$ ratio appears to be similar to that recently reported for $\mathrm{GO} / \mathrm{SiC}$ NWs foam composites, developed for photocatalytic and thermal-management applications ${ }^{9}$. Furthermore, the intensity of the $2 \mathrm{D}$ band is significantly lower than the $\mathrm{G}$ band. The estimated average of the $2 \mathrm{D}$ to $\mathrm{G}$ band ratio $\left(I_{2 \mathrm{D}} / I_{\mathrm{G}}\right)$ is $0.80 \pm 0.05$, indicating the presence of a thick GC layer. The presence of multilayer GC is further confirmed by the high-resolution TEM analysis shown later in the manuscript.

The D and G band of the GC layers in Fig.3 partially overlap with each other, suggesting the possible presence of oxygen in the graphitic system. This result seems to be consistent with the EDX results presented in Fig. 2, which indicated an approximate 10 weight $\%$ increase of oxygen after graphitization. It is also evident that the $\mathrm{G}$ band is asymmetric in shape and exhibits two shoulder peaks (Supporting information, Fig. S1). One of the peaks located around $\sim 1614 \mathrm{~cm}^{-1}$ is generally known as the $\mathrm{D}^{\prime}$ band. Both the $\mathrm{D}$ and $\mathrm{D}^{\prime}$ peaks are indicative of structural disorder in the $\mathrm{sp}^{2}$ network of carbon. The second peak known as the D"-band is noted at $1500 \mathrm{~cm}^{-1}$ and is related to graphenic edges/discontinuities ${ }^{30}$.

The inset displays three distinct peaks in the range of $1800-2300 \mathrm{~cm}^{-1}$ that are typically much lower in intensity when compared to the other characteristic Raman peaks of the GC and are related to the turbostratic stacking of the graphitic layers. These peaks located at $1885 \mathrm{~cm}^{-1}$, $2024 \mathrm{~cm}^{-1}$ and $2220 \mathrm{~cm}^{-1}$ arise from the combination of iTA+LO, LO+LA and iTO+iTA modes, respectively, where iTA, iTO, LO, LA stand for in-plane transverse acoustic, in-plane transverse optic, longitudinal optic and longitudinal acoustic modes, respectively. These results are in line with the Raman spectra of turbostratic carbon peaks reported by several groups in the past. $^{31-33}$ 

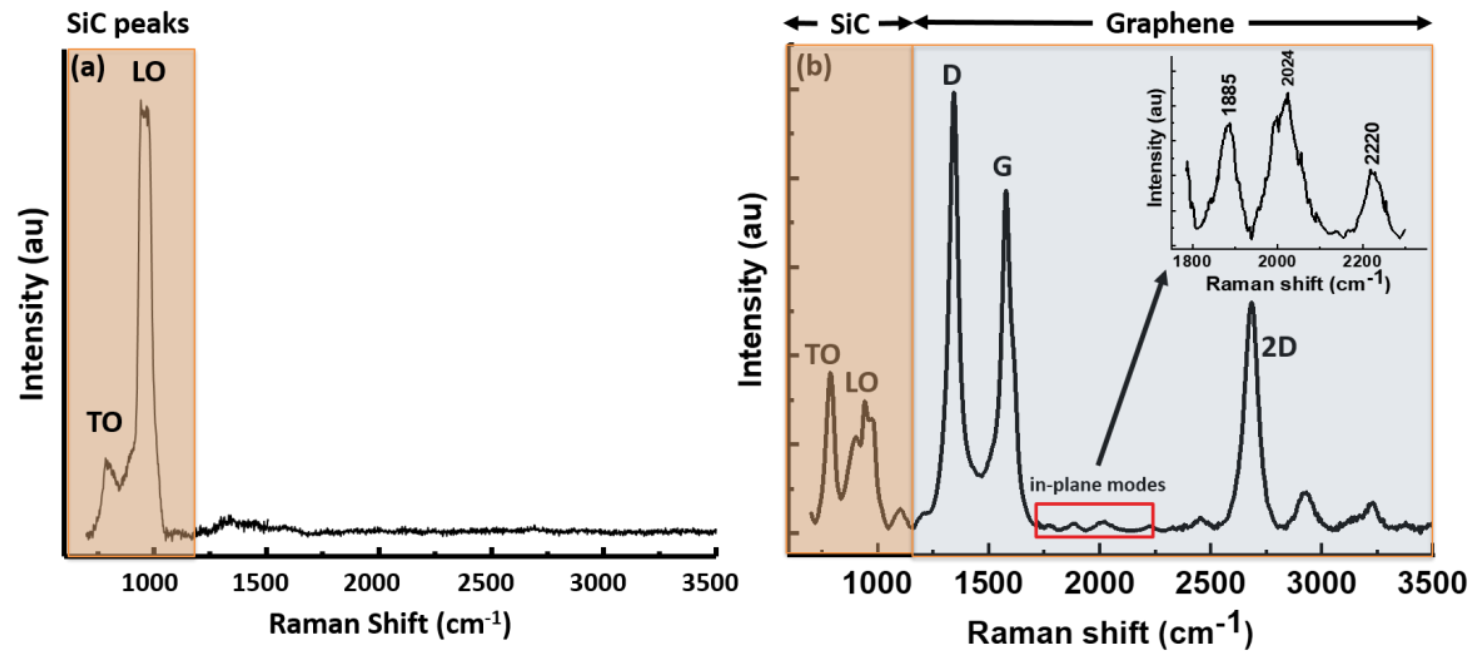

FIG. 3. Raman spectra of $3 \mathrm{C}$-SiC NWs grown on silicon (a) Before, and (b) After alloy-mediated catalytic graphitization. Before graphitization, Raman spectra of NWs show characteristic TO and LO peaks of 3C-SiC. After graphitization, additional D, G and 2D peaks appear respectively at $1340 \mathrm{~cm}-1,1578 \mathrm{~cm}-1$ and $2684 \mathrm{~cm}-1$, indicating growth of graphitic layers. Detailed investigation of Raman spectra between $1800-2300 \mathrm{~cm}-1$ displayed in inset reveals three peaks located at $1885 \mathrm{~cm}-1,2024 \mathrm{~cm}^{-1}$ and $2220 \mathrm{~cm}^{-1}$, indicating turbostratic stacking.

\section{FTIR}

Figure 4 compares the normalized FTIR spectrum of as-grown and graphitized 3C-SiC NWs measured using a Thermo Scientific Nicolet 6700 ATR-FTIR spectrometer in the range of 400$4000 \mathrm{~cm}^{-1}$. The dotted line in the figure represents the FTIR spectrum of the bare 3C-SiC NWs. The faint peak observed at $794 \mathrm{~cm}^{-1}$ corresponds to the TO mode of cubic SiC. The average FTIR spectrum of the graphitized NWs is shown as a solid line, where the TO mode of 3C-SiC at $794 \mathrm{~cm}^{-1}$ is observed again, as well as a faint peak around $\sim 970-980 \mathrm{~cm}^{-1}$, attributed to the LO mode of the cubic SiC. An additional significant peak is found around $1060 \mathrm{~cm}^{-1}$, which we ascribe to the generation of $\mathrm{C}-\mathrm{O}-\mathrm{C}$ stretching vibrations of epoxy groups ${ }^{34}$, beneficial for the functionalization of biomolecules ${ }^{2,35}$. This result confirms the incorporation of oxygen atoms in the graphitic system. Finally, it is worth noting that FTIR spectroscopy seems to yield systematically stronger information from the SiC NWs (LO and TO peaks) once the nanowires are coated with the GC layers. 


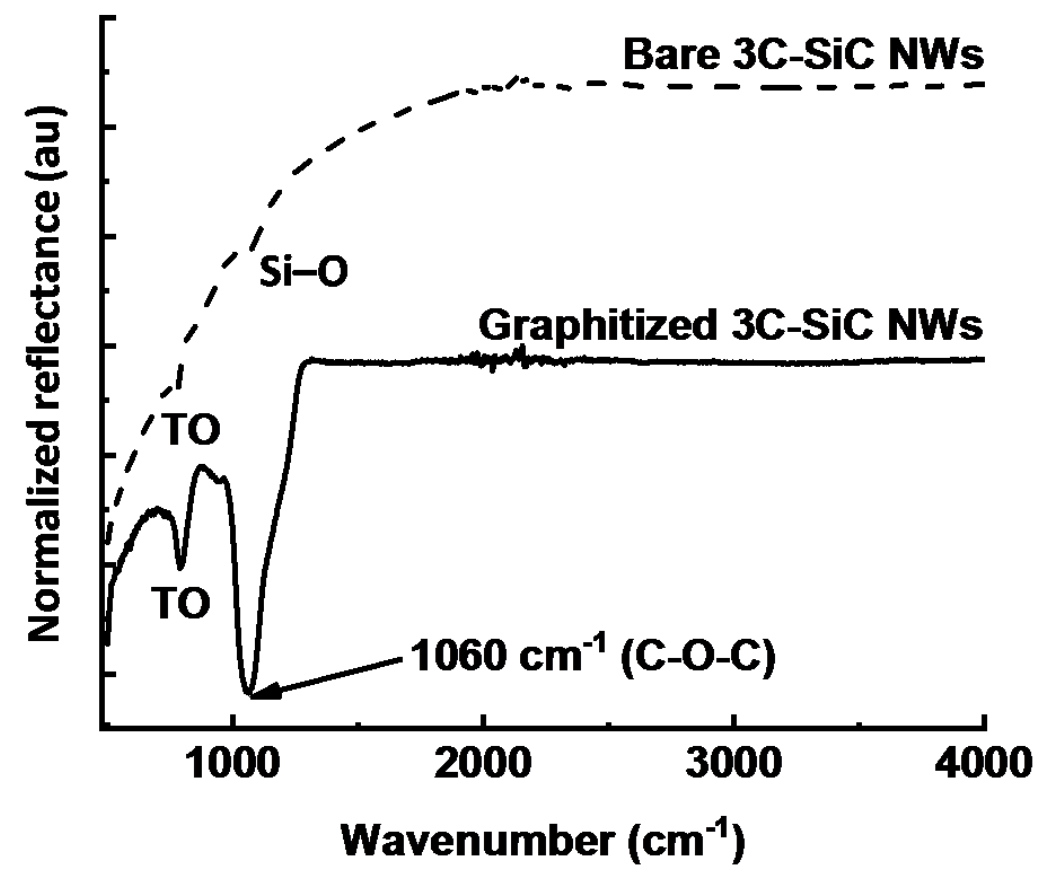

FIG. 4. FTIR spectra of bare and graphitized 3C-SiC NWs. The peak appearing at $1060 \mathrm{~cm}^{-1}$ in the graphitized sample is attributed to epoxy groups attached to the graphitic carbon layers.

\section{TEM}

Figure 5(a) shows a TEM micrograph of the as-grown 3C-SiC NWs. The NWs are approximately $\sim 30 \mathrm{~nm}$ wide, comparable to the NWs size measured via SEM imaging [Fig. 2(a)]. The SiC crystalline lattice is characterised by the presence of stacking faults on (111) planes perpendicular to the NW axis ${ }^{36}$, as shown in the high-resolution TEM image, Fig. 5(b). Figure 5(c) shows a representative TEM image of the NW sample after the graphitization process. A higher resolution TEM image [Fig. 5(d)] is acquired of the dashed squared region revealing the formation of multilayer GC with approximately $\sim 0.45 \mathrm{~nm}$ interlayer spacing, substantially larger than the thickness of mechanically exfoliated bilayer graphene $(\sim 0.34 \mathrm{~nm})$. A somewhat larger interlayer spacing was observed already for graphene grown on 3C-SiC hetero-epitaxial layers via the alloy-mediated graphitization process. ${ }^{22,}{ }^{23}$ The significantly 
larger interlayer spacing of the GC grown on $\mathrm{SiC}$ nanowires is attributed to the combined effect of turbostratic stacking as well as the presence of epoxy groups in the graphenic layers, although such spacing is still smaller than that of GO layers. ${ }^{37}$ Eight to ten graphitic layers can be observed on the NWs surface.
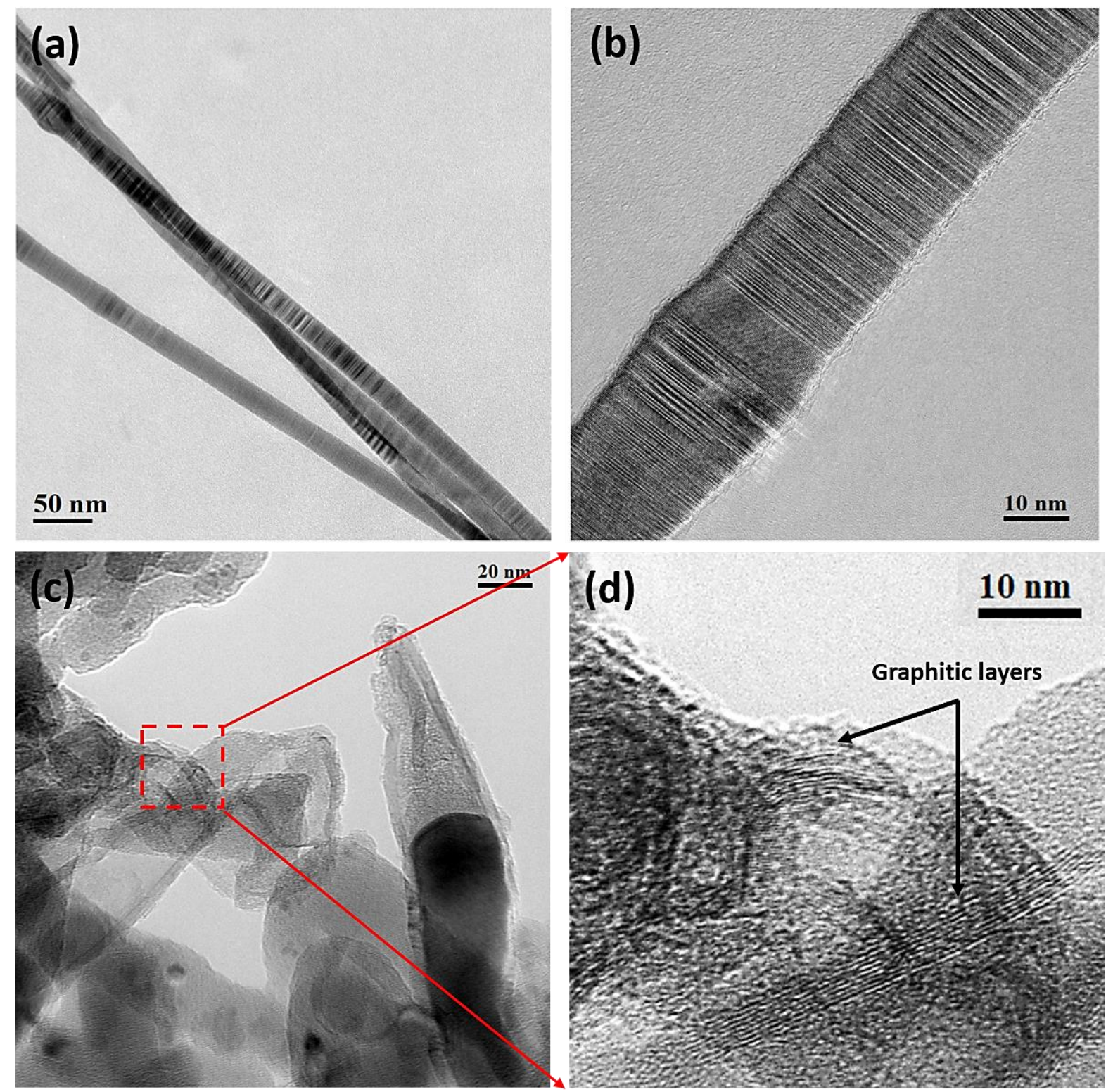

FIG. 5. a) Low resolution, and b) high resolution TEM image of as-grown 3C-SiC NWs. Stacking faults along (111) planes, perpendicular to the NW axis, are clearly seen along the wire. c) Low and d) high-resolution TEM image of graphitized SiC NWs. Eight to ten GC layers are found over the graphitized nanowires. 


\section{E. STEM-HAADF/EDX Mapping}

Figure 6(a) displays a typical STEM-HAADF image of the bare SiC NWs. Stacking faults along (111) planes, ${ }^{36}$ perpendicular to the growth direction of the nanowires, are clearly observed in the TEM images [Fig. 5(b)]. The corresponding EDX map in Fig. 6(b) shows the silicon and oxygen distribution.

STEM-HAADF images of the graphitized NWs [Fig. 6(c)] display an inner core, which appears lighter due its crystallinity, and an outer shell. For detailed elemental investigation, a high magnification EDX mapping [Fig. 6(d)] was performed in the region indicated by the square in Fig. 6(c). An approximately $\sim 15 \mathrm{~nm}$ thick oxidized layer is found around the NWs. This indicates the transformation of the $3 \mathrm{C}-\mathrm{SiC}$ NWs into a $\mathrm{SiC}$ core/ oxidized layer shell nanowire structure. Note that the formation of GC layers was not analysed by STEM-EDX elemental mapping since this technique turned out not suitable to detect such a light element as carbon with sufficient sensitivity and spatial resolution, particularly as the signal is altered by the presence of the carbon support film of the TEM grid. 

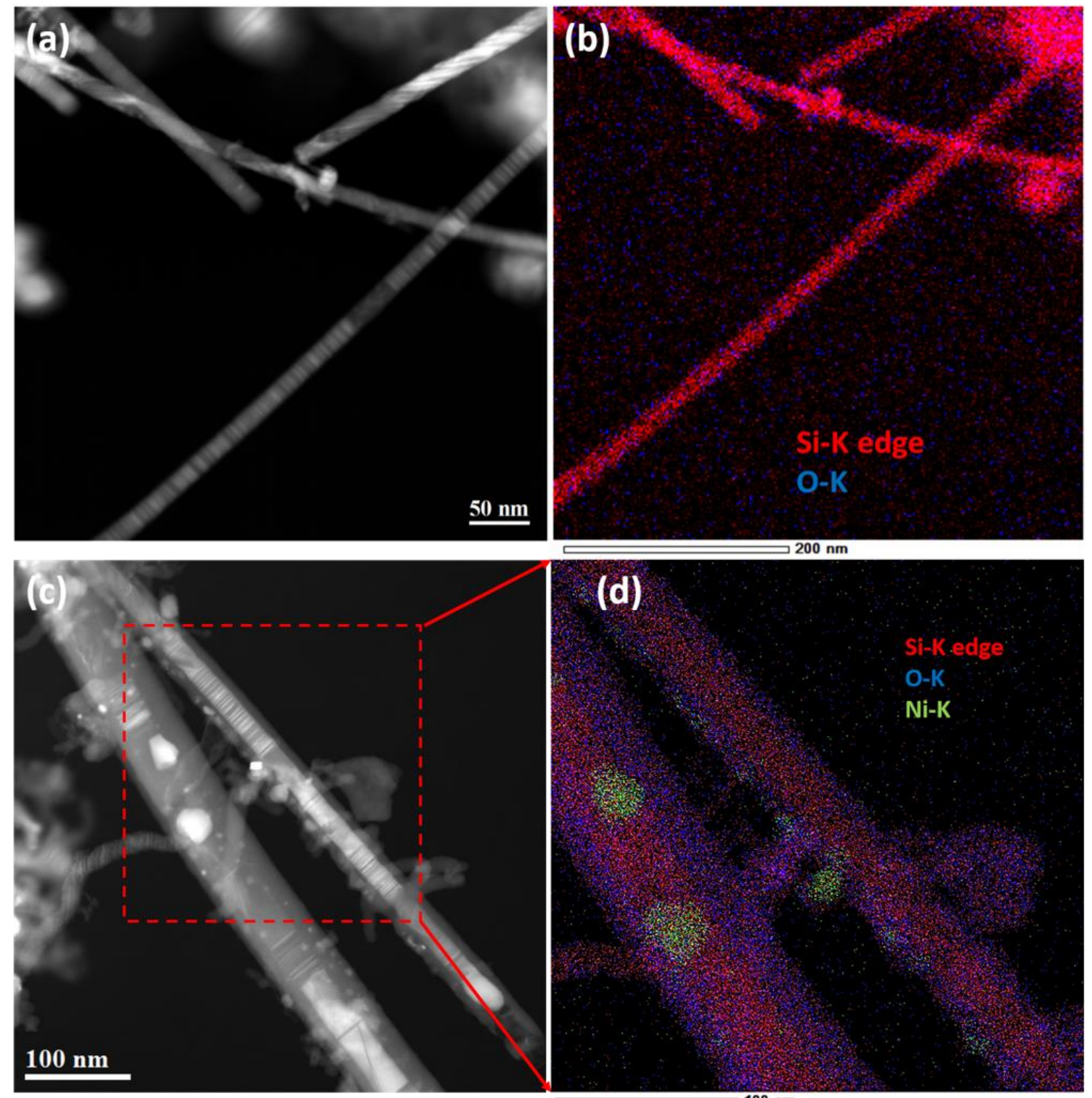

FIG. 6. a) STEM-HAADF, and b) EDX map of bare 3C-SiC NWs. c) STEM-HAADF of the graphitized NWs shows a core and shell contrast, indicating different elemental composition. d) EDX map indicates formation of a $\sim 15 \mathrm{~nm}$ thick oxidized shell layer around the 3C-SiC NWs core after graphitization. Note that we have not focused on mapping the carbon here.

\section{F. Synthesis mechanism}

The synthesis mechanism of the multilayer GC sheets around the 3C-SiC nanowires can be inferred on the basis of the presented data and our previously reported alloy-mediated graphitization process on flat $3 \mathrm{C}-\mathrm{SiC}$ surfaces $^{23}$. Fig 7 displays the proposed sequence and 
mechanism. At lower temperatures $\left(\sim 400^{\circ} \mathrm{C}\right)$, the $\sim 5 \mathrm{~nm}$ Ni layer deposited on $3 \mathrm{C}-\mathrm{SiC}$ NWs starts reacting with ambient oxygen present in the furnace forming nickel oxide (NiO). The $\mathrm{NiO} / \mathrm{Ni}$ react with the top $\sim 5 \mathrm{~nm}$ of $\mathrm{SiC}$ around the NW surface at higher temperatures (400$1050^{\circ} \mathrm{C}$ ) producing nickel silicides, oxides and releasing atomic carbon atoms into the system. ${ }^{38,}{ }^{39}$ Upon cooling, an approximately $\sim 15 \mathrm{~nm}$-thick, highly oxidized layer develops around the NWs surface coated with multilayer GC sheets [Fig. 5(d) and 6(d)]. Note that a similar $\sim 20 \mathrm{~nm}$ partly oxidized/amorphized $\mathrm{SiC}$ layer was found underneath the graphene obtained via alloy-mediated graphitization of flat $3 \mathrm{C}-\mathrm{SiC} / \mathrm{Si}$ layers ${ }^{23}$.

The Freckle solution wet etch is the final step needed for removing the silicides, excess carbon and unreacted metal catalysts, and expose the graphene. As we discussed earlier in the manuscript, the limitation on the wet etch duration for the nanowires has led to Ni residues.

The EDX maps and SEM micrographs show $\mathrm{Ni}$-containing residues in the form of droplets on the graphitized NWs surface, indicative of dewetting at elevated temperature.

Finally, we note that, given the one-dimensional structure of the NWs and their very small volume, plus the fact that our process leads to the prevalent formation of a nickel disilicide phase ( $\mathrm{NiSi}_{2}$ where each $\mathrm{Ni}$ atom consume two silicon atoms), ${ }^{23,38}$ the control of the thickness of Ni layer sputtered over SiC NWs is crucial. A too thick Ni layer can react with the entire $\mathrm{SiC}$ volume, dissolving the nanowires. In order to obtain a single graphitic layer around the NWs, an exceptionally thin and uniform Ni layer is desirable. We believe that a highly controlled $\mathrm{Ni}$ deposition technique such as atomic layer deposition (ALD) ${ }^{40}$ would offer adequate potential to serve this purpose. 


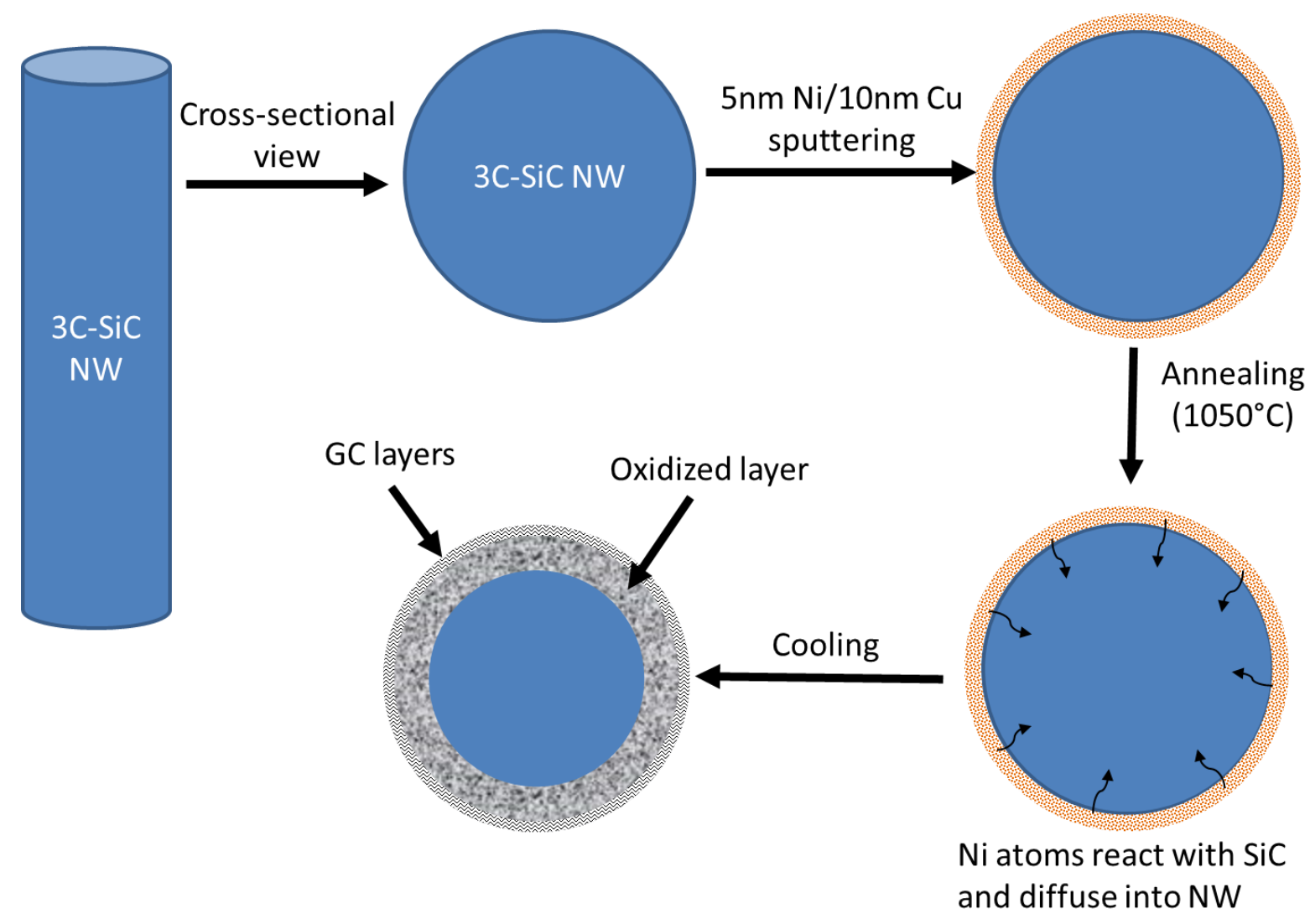

FIG. 7. Schematic of the growth of graphitic carbon layers around SiC NWs via the Ni/Cu alloy -mediated catalytic process, which involves an oxidation of the outer shell of the SiC nanowires as free carbon is released to form the GC layers.

\section{CONCLUSIONS}

In conclusion, we demonstrate a route for the synthesis of multi-layered graphitic carbon (GC) sheets around 3C-SiC nanowires. Three distinct Raman peaks appear between $1800 \mathrm{~cm}^{-1}$ and $2300 \mathrm{~cm}^{-1}$, reveal a turbostratic stacking of the GC sheets. The STEM-HAADF/EDX map indicate formation of a $\sim 15 \mathrm{~nm}$ oxidized layer around the 3C-SiC NW core. A sharp FTIR reflection peak observed at $\sim 1060 \mathrm{~cm}^{-1}$ suggest the presence of epoxy group on the graphitized NWs surface, which may provide attachment sites to a number of biological molecules including protein, DNA, RNA etc ${ }^{35,41,42}$. We attribute the large inter-layer spacing of $\sim 0.45$ $\mathrm{nm}$ to the presence of such epoxy groups, in addition to the turbostratic stacking. We believe that the surface functionalization and intercalation capabilities of the obtained GC sheets, 
combined to the exceptional mechanical properties of SiC NWs and the bio-compatibility of both materials, make these core-shell GC/SiC-NWs very promising nanostructures for biosensing applications.

\section{SUPPLEMENTARY MATERIAL}

See supplementary material for the deconvoluted Raman G- band of the graphitic layers grown on $\mathrm{SiC}$ NWs.

\section{ACKNOWLEDGEMENTS}

This work was performed in part at the University of Technology Sydney, Australia and at the IMEM-CNR Institute, Italy. Support from the Air Force Office for Scientific Research through grant AOARD 18IOA052 is also acknowledged. MB and FR acknowledge the help of Dr. Giovanni Attolini for the preparation of nanowires.

\section{REFERENCES}

${ }^{1}$ C. I. L. Justino, A. R. Gomes, A. C. Freitas, A. C. Duarte and T. A. P. Rocha-Santos, TrAC Trends in Analytical Chemistry. 91, 53-66 (2017)

${ }^{2}$ M. Pumera, Materials Today. 14, 308-315 (2011)

${ }^{3}$ J. Lee, J. Kim, S. Kim and D.-H. Min, Advanced Drug Delivery Reviews. 105, 275-287 (2016)

${ }^{4}$ X. Dong, Y. Shi, W. Huang, P. Chen and L.-J. Li, Advanced Materials. 22, 1649-1653 (2010)

${ }_{5}^{5}$. Sabury, S. H. Kazemi and F. Sharif, Materials Science and Engineering: C. 49, 297-304 (2015)

${ }^{6}$ I.-H. Lee, D. Yoo, P. Avouris, T. Low and S.-H. Oh, Nature Nanotechnology. (2019)

${ }^{7}$ K.-J. Huang, Y.-J. Liu, H.-B. Wang and Y.-Y. Wang, Electrochimica Acta. 118, 130-137 (2014)

${ }^{8}$ S. Singh, K. Rathi and K. Pal, Journal of Alloys and Compounds. 740, 436-445 (2018)

${ }^{9}$ M. Han, X. Yin, Z. Hou, C. Song, X. Li, L. Zhang and L. Cheng, ACS applied materials \& interfaces. 9, 11803-11810 (2017)

${ }^{10}$ H. Qu, Y. Wang, Y. S. Ye, W. Zhou, S. P. Bai, X. P. Zhou, H. Y. Peng, X. L. Xie and Y.-W. Mai, Journal of Materials Chemistry A. 5, 22361-22371 (2017)

${ }^{11}$ Kenry, W. C. Lee, K. P. Loh and C. T. Lim, Biomaterials. 155, 236-250 (2018)

${ }^{12} \mathrm{C}$. Wang and D. Astruc, Progress in Materials Science. 94, 306-383 (2018)

${ }^{13}$ S. E. Saddow, Silicon carbide biotechnology: a biocompatible semiconductor for advanced biomedical devices and applications (Elsevier, 2012)

${ }^{14}$ A. R. Kermany, J. S. Bennett, V. M. Valenzuela, W. P. Bowen and F. Iacopi, physica status solidi (a). 214, 1600437 (2017)

${ }^{15}$ A. R. Kermany, G. Brawley, N. Mishra, E. Sheridan, W. P. Bowen and F. Iacopi, Applied Physics Letters. 104, 081901 (2014)

${ }^{16}$ L. Fradetal, E. Bano, G. Attolini, F. Rossi and V. Stambouli, Nanotechnology. 27, 235501 (2016) 
${ }^{17}$ E. Bano, L. Fradetal, M. Ollivier, J.-H. Choi and V. Stambouli, Chapter 9 - SiC Nanowire-Based Transistors for Electrical DNA Detection (Elsevier, 2016) p.261-310

${ }^{18}$ A. Cacchioli, F. Ravanetti, R. Alinovi, S. Pinelli, F. Rossi, M. Negri, E. Bedogni, M. Campanini, M. Galetti, M. Goldoni, P. Lagonegro, R. Alfieri, F. Bigi and G. Salviati, Nano Letters. 14, 4368-4375 (2014)

${ }^{19}$ P. Lagonegro, F. Rossi, C. Galli, A. Smerieri, R. Alinovi, S. Pinelli, T. Rimoldi, G. Attolini, G. Macaluso, C. Macaluso, S. E. Saddow and G. Salviati, Materials Science and Engineering: C. 73, 465-471 (2017) ${ }^{20}$ O. Cooper, B. Wang, C. L. Brown, J. Tiralongo and F. lacopi, IEEE Access. 4, 477-497 (2016)

${ }^{21}$ B. V. Cunning, M. Ahmed, N. Mishra, A. R. Kermany, B. Wood and F. lacopi, Nanotechnology. 25, 325301 (2014)

${ }^{22}$ F. lacopi, N. Mishra, B. V. Cunning, D. Goding, S. Dimitrijev, R. Brock, R. H. Dauskardt, B. Wood and J. Boeckl, J. Mater. Res. 30, 609-616 (2015)

${ }^{23}$ N. Mishra, J. J. Boeckl, A. Tadich, R. T. Jones, P. J. Pigram, M. Edmonds, M. S. Fuhrer, B. M. Nichols and F. lacopi, Journal of Physics D: Applied Physics. 50, 095302 (2017)

${ }^{24}$ M. Negri, S. C. Dhanabalan, G. Attolini, P. Lagonegro, M. Campanini, M. Bosi, F. Fabbri and G. Salviati, CrystEngComm. 17, 1258-1263 (2015)

${ }^{25}$ F. Fabbri, F. Rossi, M. Negri, R. Tatti, L. Aversa, S. C. Dhanabalan, R. Verucchi, G. Attolini and G. Salviati, Nanotechnology. 25, 185704 (2014)

${ }^{26} \mathrm{~N}$. Mishra, J. Boeckl, N. Motta and F. lacopi, physica status solidi (a). 213, 2269-2269 (2016)

${ }^{27}$ P. A. Temple and C. Hathaway, Physical Review B. 7, 3685 (1973)

${ }^{28}$ S.-i. Nakashima and H. Harima, physica status solidi (a). 162, 39-64 (1997)

${ }^{29}$ Z. Feng, W. Choyke and J. Powell, Journal of applied physics. 64, 6827-6835 (1988)

${ }^{30} \mathrm{M}$. Ahmed, B. Wang, B. Gupta, J. J. Boeckl, N. Motta and F. lacopi, Journal of The Electrochemical Society. 164, A638-A644 (2017)

${ }^{31}$ R. Rao, R. Podila, R. Tsuchikawa, J. Katoch, D. Tishler, A. M. Rao and M. Ishigami, ACS nano. 5, 15941599 (2011)

32J. A. Garlow, L. K. Barrett, L. Wu, K. Kisslinger, Y. Zhu and J. F. Pulecio, Scientific reports. 6, 19804 (2016)

${ }^{33}$ A. Niilisk, J. Kozlova, H. Alles, J. Aarik and V. Sammelselg, Carbon. 98, 658-665 (2016)

${ }^{34} \mathrm{~K}$. Haubner, J. Murawski, P. Olk, L. M. Eng, C. Ziegler, B. Adolphi and E. Jaehne, ChemPhysChem. 11, 2131-2139 (2010)

${ }^{35}$ D. P. Singh, C. E. Herrera, B. Singh, S. Singh, R. K. Singh and R. Kumar, Materials Science and Engineering: C. 86, 173-197 (2018)

${ }^{36}$ F. lacopi, G. Walker, L. Wang, L. Malesys, S. Ma, B. V. Cunning and A. lacopi, Applied Physics Letters. 102, 011908 (2013)

${ }^{37}$ B. Rezania, N. Severin, A. V. Talyzin and J. r. P. Rabe, Nano letters. 14, 3993-3998 (2014)

${ }^{38}$ B. A. Julies, D. Knoesen, R. Pretorius and D. Adams, Thin Solid Films. 347, 201-207 (1999)

${ }^{39}$ Z.-Y. Juang, C.-Y. Wu, C.-W. Lo, W.-Y. Chen, C.-F. Huang, J.-C. Hwang, F.-R. Chen, K.-C. Leou and C.-

H. Tsai, Carbon. 47, 2026-2031 (2009)

${ }^{40}$ M. M. Kerrigan, J. P. Klesko, K. J. Blakeney and C. H. Winter, ACS Applied Materials \& Interfaces. 10, 14200-14208 (2018)

${ }^{41}$ N. D. Q. Chau, G. Reina, J. Raya, I. A. Vacchi, C. Ménard-Moyon, Y. Nishina and A. Bianco, Carbon. 122, 643-652 (2017)

${ }^{42}$ V. Georgakilas, M. Otyepka, A. B. Bourlinos, V. Chandra, N. Kim, K. C. Kemp, P. Hobza, R. Zboril and K. S. Kim, Chemical reviews. 112, 6156-6214 (2012) 


\section{SUPPLEMENTARY MATERIAL}

Figure S1 shows Raman spectra of GC between $1100 \mathrm{~cm}^{-1}$ to $1800 \mathrm{~cm}^{-1}$. The Raman fitting below shows a more detailed analysis of the G and D bands, evidencing the presence of two additional vibrations: the D' vibration, at $1615 \mathrm{~cm}^{-1}$, related to structural disorder in the $\mathrm{sp}^{2}$ network of carbon and the D" vibration, around $1500 \mathrm{~cm}^{-1}$, attributed to the presence of a small component of ribbon -like edges.

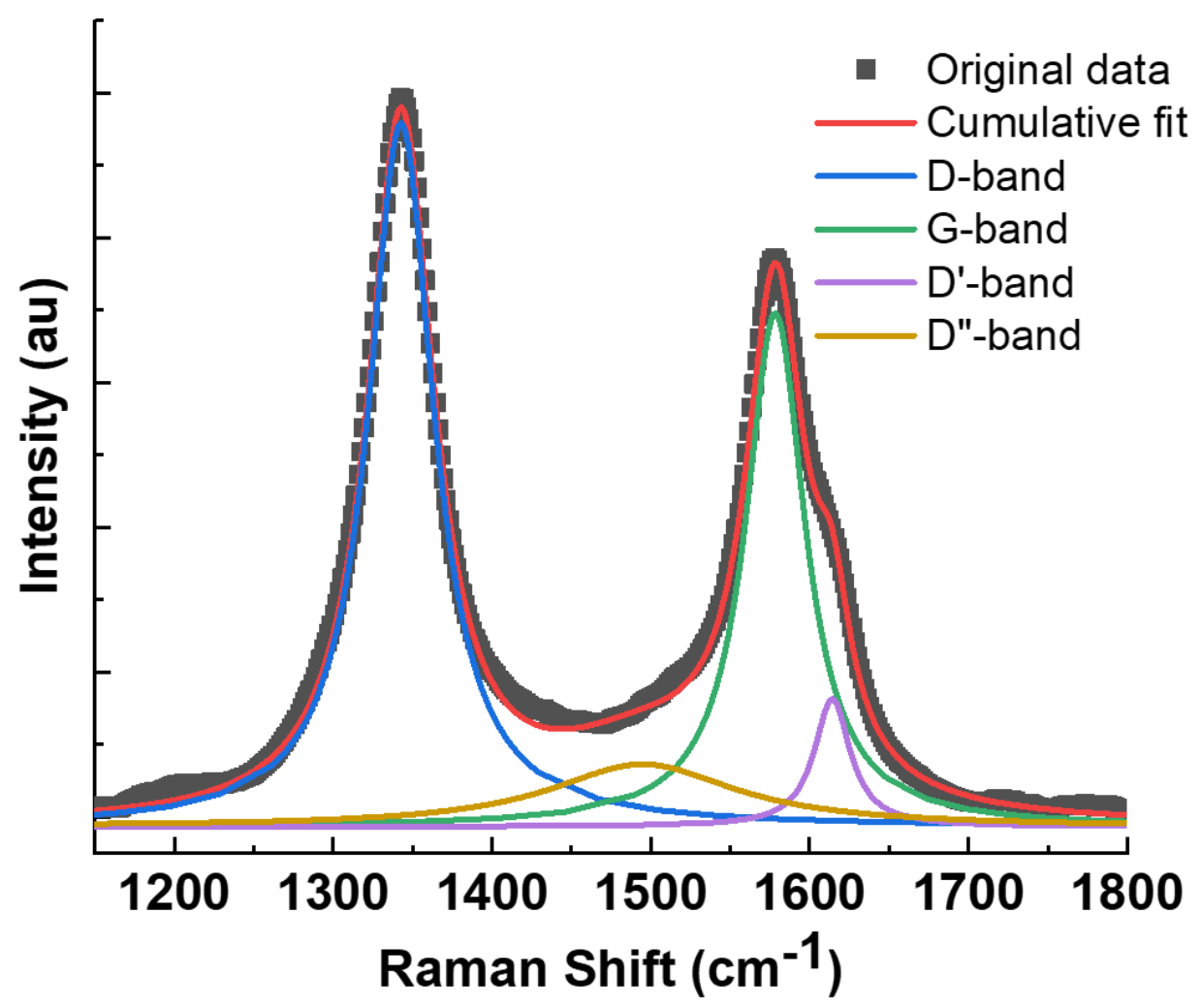

FIG S1: Raman spectra of GC between $1100 \mathrm{~cm}-1$ to $1400 \mathrm{~cm}-1$ can be deconvoluted in four peaks: D-, G-, D'and D"-band. 